50 (2019) pp. 89-92

DOI: $10.33039 / \mathrm{ami} .2019 .03 .002$

http://ami.uni-eszterhazy.hu

\title{
Algorithm for the generation of complement-free sets*
}

\author{
Dániel Fülöp, Carolin Hannusch \\ Faculty of Informatics, University of Debrecen, Hungary \\ fulop.daniel9623@gmail.com \\ hannusch.carolin@inf .unideb.hu \\ Submitted: February 6, 2019 \\ Accepted: March 29, 2019 \\ Published online: April 6, 2019
}

\begin{abstract}
We introduce an algorithm for the generation of complement-free sets of binary $m$-tuples, where $m$ is even. We also provide an implementation for this algorithm for $m=12$. Such complement-free sets are needed for the generation of a new class of error-correcting codes, which were introduced by Hannusch and Lakatos. These codes build the fundamental improvement in the cryptographic system of Dömösi, Hannusch and Horváth. Therefore the generation of complement-free sets will be important for cryptographic applications. In the end of the paper we give some interesting facts about complement-free sets as combinatorial objects.
\end{abstract}

Keywords: algorithmic computation, discrete sets

MSC: 03D32, 97N70

\section{Introduction and notation}

Let $m$ be an even number, thus $m=2 k$ for some $k \in \mathbb{N}$. Then let $X$ be the set of all binary $m$-tuples with exactly $k$ pieces of 1 -s and $k$ pieces of 0 -s.

Definition 1.1. Let $x \in X$ be an arbitrary element. Further we denote the whole-1 tuple of length $m$ by $\mathbf{1}$. Then we say that $\mathbf{1}-x$ is the complement of $x$.

*This work was supported by the construction EFOP-3.6.3-VEKOP-16-2017-00002. The project was supported by the European Union, co-financed by the European Social Fund. 
Definition 1.2. Let $Y \subset X$, such that $y \in Y$ implies $\mathbf{1}-y \notin Y$. Then $Y$ is called complement-free subset of $X$. If $Y$ has order $\frac{1}{2}\left(\begin{array}{c}m \\ k\end{array}\right)$, then we say that $Y$ is a maximal complement-free subset.

In this paper, we give an algorithm for generating a maximal complement-free set randomly. Such sets are used in [3] for the construction of self-dual errorcorrecting codes of length $2^{m}$ and with minimum distance $2^{k}$. These codes are called HL-codes and they are used in the cryptographic system of Dömösi, Hannusch and Horváth in [1]. In order to develop an effective implementation of the DHHcryptosystem [2], it is necessary to generate a complement-free set effectively.

The DHH-crypotosystem is using the HL-code for $m=12$, therefore we provide an implementation of our algorithm for $m=12$ in $\mathrm{C}++$ under the following link:

https://arato.inf.unideb.hu/hannusch.carolin/alg.cpp

\section{The algorithm}

We fix $m=2 k$.

Input: number $l$ with $0 \leq l \leq \frac{1}{2}\left(\begin{array}{c}m \\ k\end{array}\right)-1$

Output: maximal complement-free set $Y$

Step 1:

- Let $A$ be the list of all binary $m$-tuples with $k$ pieces of 1 -s, where the first coordinate is 1 .

- Let $B$ be the list of all binary $m$-tuples where $B[i]=\mathbf{1}-A[i]$.

Step 2: for $i$ from 1 to $\frac{1}{2}\left(\begin{array}{c}m \\ k\end{array}\right)+l-1 \bmod \frac{1}{2}\left(\begin{array}{c}m \\ k\end{array}\right)$ do

$i:=0$ or 1 randomly; end for;

Step 3: if $i=0$ then $Y[i]:=A[i]$; else $Y[i]:=B[i]$. end for;

Continue Step 2 until $\operatorname{order}(Y)=\frac{1}{2}\left(\begin{array}{c}m \\ k\end{array}\right)$.

This algorithm provides one possibility to create a complement-free set. Further research step will be the use of this algorithm (esp. the implementation) in an implementation of the DHH-cryptosystem. A fast algorithm with low memoryneed is a necessary part of a competetive DHH-cryptosystem. The provided algorithm generates 100 complement-free sets of order 462 in 2.7 seconds and 1000 complement-free sets of order 462 in 15.8 seconds on $\operatorname{Intel}(R)$ Core(TM)2 Duo CPU at $2.93 \mathrm{GHz}$. 


\section{Additional facts about complement-free sets}

The ordering of the list $A$ in Step 1 of the algorithm introduced in Section 2 should be kept secret. This will improve the security of the algorithm when it is used in Cryptography. For $m=12$ the list $A$ has 462 elements, which means there are 462 ! possible orders of the elements of $A$ and since

$$
462 !>10^{1032}
$$

this cannot be brute-forced.

So, let us now assume that $A$ is secret. For the random value of $i$ in Step 2 of the algorithm we need a random generator with almost $50 \%$ possibility that if $i=0$, then $i+1=1$ and vice versa. Applying such a random generator we have a probability of $\left(\frac{1}{2}\right)^{462}$ that we generate the same complement-free set twice. A good random generator can be found e.g. in [4].

Some more interesting things can be investigated in relation to complement-free sets if we have a more detailed look at one set itself. Given a complement-free set $Y$, each element $y \in Y$ consists of $m$ coordinates. We will count the 1 -s in a fixed coordinate for all $y \in Y$. For example, let $Y=\{(1,1,0,0),(1,0,0,1),(0,1,0,1)\}$. Then we have two 1-s in each four positions. Thus we will say that $Y$ is of type $(2,2,2,2)$ according to the following definition:

Definition 3.1. We say that the complement-free set $Y$ is of type $\nu=\left(n_{1}, \ldots, n_{m}\right)$, if

$$
n_{i}=\sum_{y \in Y} y_{i},
$$

i.e. $n_{i}$ is the number of 1 -s in the $i$-th coordinate of all binary strings in $Y$.

Remark 3.2. We have $\sum_{i=1}^{m} n_{i}=k \cdot \frac{1}{2}\left(\begin{array}{c}m \\ k\end{array}\right)$.

Let us denote $\sum_{i=1}^{m} n_{i}$ by $N$. Then it is clear, that if $\nu$ is the type of a complement-free set, then $\nu$ is also a partition of $N$. This statement is not true in the other way, since e.g. for $m=6$ we have $N=30$ and $(7,7,5,3,3,1)$ is a partition, but there is no complement-free set of such a type.

Proposition 3.3. For fix $m=2 k$ there exist at least $\frac{1}{4}\left(\begin{array}{c}m \\ k\end{array}\right)+1$ different types of complement-free sets.

Proof. We may assume $n_{1} \geq n_{2} \geq \cdots \geq n_{m}$. Then there exists exactly one type with $n_{1}=\frac{1}{2}\left(\begin{array}{c}m \\ k\end{array}\right)$ (namely the complement-free set consists of all elements of the list $A$ in this case). Now imagine, that we change one element of the set from $A[i]$ to $B[i]$. Thus the new complement-free set has type $n_{1}=\frac{1}{2}\left(\begin{array}{c}m \\ k\end{array}\right)-1$. We continue this step until the descending order $n_{1} \geq n_{2} \geq \cdots \geq n_{m}$ can be fulfilled. Since $k \cdot \frac{1}{2}\left(\begin{array}{c}m \\ k\end{array}\right)$ is divisible by $m$ there exists exactly one type with $n_{1}=\frac{1}{4}\left(\begin{array}{c}m \\ k\end{array}\right)$.

Computations of all types of complement-free sets for small values of $m$ let us conjecture that the distribution of types with $\frac{1}{4}\left(\begin{array}{c}m \\ k\end{array}\right) \leq n_{1} \leq \frac{1}{2}\left(\begin{array}{c}m \\ k\end{array}\right)$ is close to Gaussian distribution. Further, it turns out that computing all types of complement-free 
sets for $m=8$ needs a lot of computation and cannot be done fast. Thus we come to the following open problems.

Problem 3.4. Determine all types of complement-free sets for fix m!

Problem 3.5. Show the distribution of complement-free sets with respect to the largest value in the type! (Is it Gaussian distribution?)

\section{References}

[1] P. Dömösi, C. Hannusch, G. Horváth: A cryptographic system based on a new class of binary error-correcting codes, submitted.

[2] P. Dömösi, C. Hannusch, G. Horváth: Public key cryptographic method and apparatus for data encryption and decryption based on error-correcting codes, Budapest: Hungarian Intellectual Property Office, patent application, P1800038, 2018.

[3] C. Hannusch, P. Lakatos: Construction of self-dual binary $\left[2^{2 k}, 2^{2 k-1}, 2^{k}\right]$-codes, Algebra and Discrete Mathematics 21.1 (2016), pp. 59-68.

[4] T. Herendi: Construction of uniformly distributed linear recurring sequences modulo powers of 2, Uniform distribution theory 13.1 (2018), pp. 109-129, DOI: 10.1515/udt-2018-0006. 